\title{
Adatom-induced lateral inhomogeneity of quantum well states in metal multilayers
}

\author{
Udo Schwingenschlögl, ${ }^{1, *}$ Takashi Uchihashi, ${ }^{2}$ Cono Di Paola, ${ }^{1}$ and Richard Berndt ${ }^{3}$ \\ ${ }^{1}$ PSE Division, KAUST, Thuwal 23955-6900, Kingdom of Saudi Arabia \\ ${ }^{2}$ International Center for Materials Nanoarchitectonics, National Institute for Materials Science, 1-1 Namiki, \\ Tsukuba, Ibaraki 305-0044, Japan \\ ${ }^{3}$ Institut für Experimentelle und Angewandte Physik, Christian-Albrechts-Universität zu Kiel, D-24098 Kiel, Germany
}

(Received 3 June 2010; published 13 July 2010)

\begin{abstract}
The influence of $\mathrm{Co}$ adatoms on the quantum well states (QWSs) existing in $\mathrm{Cu} / \mathrm{Co}(100)$ multilayers is investigated by means of ab initio calculations. The typical oscillations of the density of states at the Fermi level as a function of the number of $\mathrm{Cu}$ layers are found to be strongly perturbed by the presence of adatoms on the surface. In a lateral direction, the QWSs exhibit atomic-scale variations, which depend on the number of $\mathrm{Cu}$ layers. These results suggest that the phase accumulation model, which is often used for analyzing QWS, is not sufficient to interpret electronic features near adatoms and call for experimental real-space investigations of QWS.
\end{abstract}

DOI: 10.1103/PhysRevB.82.033406

PACS number(s): 73.21.-b, 73.61.At

Thin layers of crystalline metals may exhibit quantum wells states (QWSs). These states can be understood in terms of an interference of electron waves reflected at the interfaces of the film to the substrate and to the vacuum. Beyond being an appealing manifestation of the wave properties of electrons in metals, QWSs are vital for technologically relevant phenomena such as giant magnetoresistance in magnetic multilayers or two-dimensional electron gases in semiconductor heterostructures. ${ }^{1-3}$ Many physical properties of QWS can be understood within the framework of the phase accumulation model, which considers the reflections and phase shifts of electron waves at the interfaces. ${ }^{4-6}$

So far, studies on QWS focused on laterally homogeneous systems. One reason for this state of affairs may be that photoelectron spectroscopy, which is the most widely used experimental technique for studying QWS, ${ }^{7-9}$ usually does not provide a nanoscale lateral resolution. However, local variations of QWSs are of concern as soon as nanoscale structures are present. ${ }^{10}$ This issue plays a crucial role when QWSs are used to manipulate the electronic or magnetic properties of molecules or atoms. ${ }^{11,12}$ Indeed, the Kondo effect observed from Mn-phtalocyanine molecules or Co adatoms was successfully modulated using QWS in $\mathrm{Pb} / \mathrm{Si}(111)$ or $\mathrm{Cu} / \mathrm{Co}(100)$ layers. ${ }^{13-15}$ In discussing these experiments, a countereffect of adsorbed molecules or atoms on the QWS was tentatively suggested. ${ }^{13,14,17}$ A prediction of the phase shift of electron waves at locally perturbed boundaries, however, is beyond the scope of the phase accumulation model. In fact, the presence of well-defined wave vectors and phase shifts needs to be reconsidered when lateral inhomogeneities are present. For these reasons, it is desirable to extend $a b$ initio calculations, which have shown to accurately reproduce QWS in a variety of homogeneous systems ${ }^{16-20}$ to scenarios with a lowered symmetry.

This Brief Report addresses the influence of Co adatoms on the density of states (DOS) of QWS in $\mathrm{Cu} / \mathrm{Co}(100)$ multilayers by means of first-principles calculations. In the absence of adatoms, the variation of the DOS at the Fermi level $\left(E_{F}\right)$ as a function of the number of $\mathrm{Cu}$ layers can reasonably be fitted using the phase accumulation model with two quantum oscillations. ${ }^{9,13,21}$ In contrast, the DOS of a surface which is perturbed by a 1/4 monolayer (ML) of Co adatoms is drastically modified and an analysis within the phase accumulation model proves to be inadequate. Lateral variations of the DOS are found and analyzed for $\mathrm{Cu}$ slabs covered with a $1 / 9 \mathrm{ML}$ of Co adatoms. The spatial coherence of the oscillations is found to depend on the number of $\mathrm{Cu}$ layers.

Our results are based on electronic structure calculations within density-functional theory. More specifically, we apply the augmented spherical wave approach ${ }^{22}$ and the localdensity approximation. ${ }^{23}$ An important advantage of the augmented spherical wave approach is its minimal basis set, which enables calculations for huge supercells as required for the present study. As valence states we take into consideration the $\mathrm{Co} / \mathrm{Cu} 4 s, 4 p$, and $3 d$ orbitals and complement this basis set by orbitals of additional augmentation spheres which account for the voids in the crystal structure. Brillouin-zone integrations are performed using the linear tetrahedron method. The number of $k$ points was increase until convergence was reached. In all our calculations this has been the case for at most $500 k$ points within the irreducible wedge of the supercell Brillouin zone. The $\mathrm{Cu}(001)$ slab is represented by a supercell built from fcc $\mathrm{Cu}$ by stacking a variable number of fcc unit cells along the [001] direction. Moreover, a Co substrate can be modeled by continuing the fcc $\mathrm{Cu}$ lattice by $\mathrm{Co}$ atoms. ${ }^{24}$ However, in the $a b$ plane, which is the interface plane, the Co lattice constant has to be adapted to the $\mathrm{Cu}$ value in order to avoid a lattice mismatch. On the $\mathrm{Cu}(001)$ surface, the $\mathrm{Co}$ adatoms are assumed to occupy the hollow sites and the distance to the $\mathrm{Cu}$ surface layer is set to the optimized value of $1.62 \AA .{ }^{25}$

Figure 1(a) (solid line) shows the calculated DOS at $E_{F}$ averaged at the top $\mathrm{Cu}$ layer as a function of the number of $\mathrm{Cu}$ layers $\left(N_{\mathrm{Cu}}\right)$ in pure $\mathrm{Cu}$ slabs. The DOS oscillates with a periodicity of about 2.5 atomic layers, while its amplitude decreases gradually with increasing $N_{\mathrm{Cu}}$. This behavior can be ascribed to quantum oscillation. The electron waves within a confined space constructively or destructively interfere depending on the total width of the $\mathrm{Cu}$ slab, which defines the traveling length for electrons. The periodicity of the oscillation is not determined by the Fermi wavelength but, in the case of $\mathrm{Cu}(100)$ layers, rather by two reciprocal length 


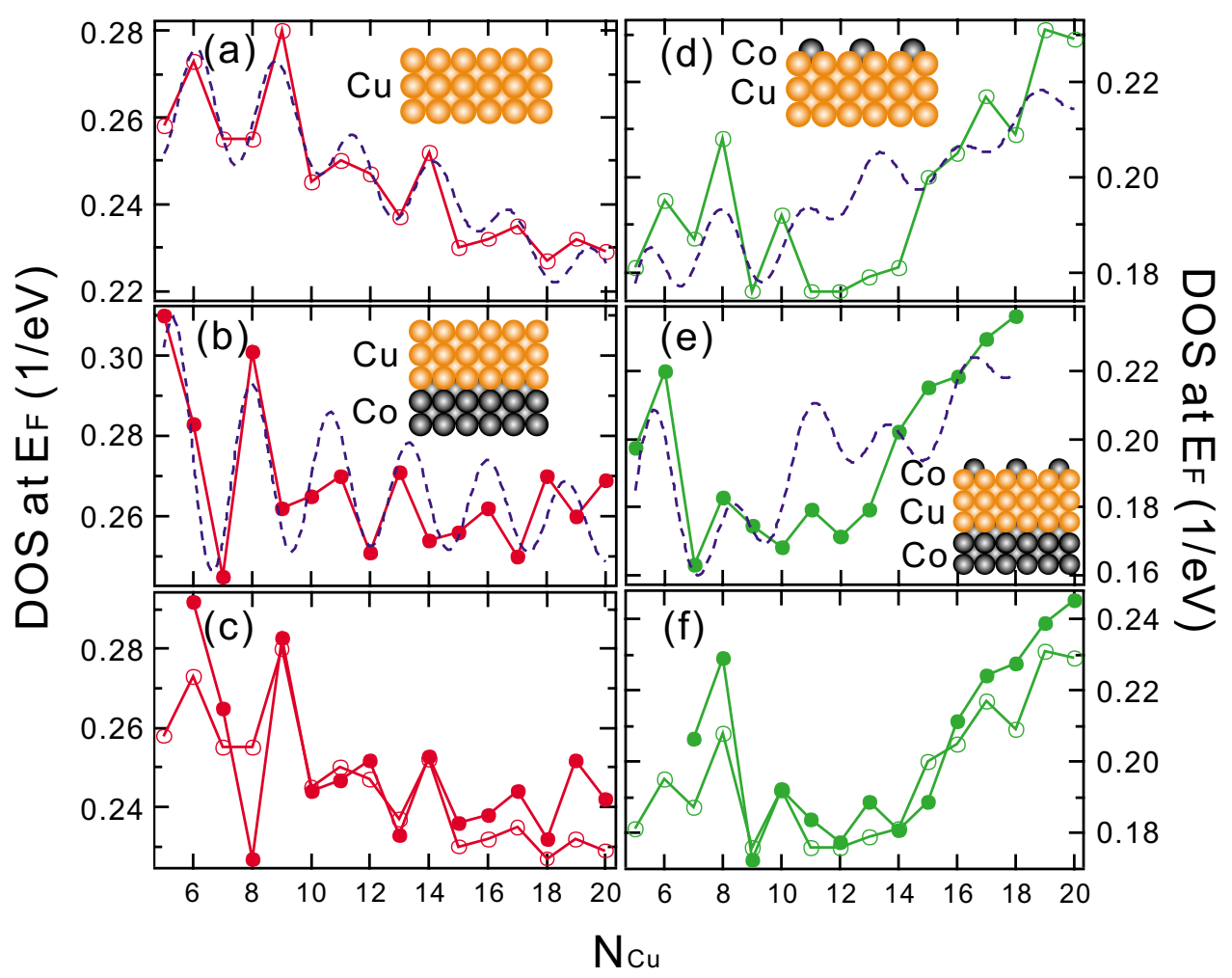

FIG. 1. (Color online) Averaged DOS at $E_{F}$ for the top $\mathrm{Cu}$ layer as a function of the number of Cu layers $\left(N_{\mathrm{Cu}}\right)$ in $\mathrm{Cu} / \mathrm{Co}$ slabs obtained by ab initio calculations (solid lines) and fitting results using Eq. (1) (dashed lines). (a) Pure Cu slabs. (b) Cu slabs on a Co substrate. (c) Comparison of (a) (open circles) and (b) (closed circles). The data of (b) are offset by one layer along the abscissa and by $-0.018 \mathrm{eV}^{-1}$ along the ordinate. (d) Pure $\mathrm{Cu}$ slab covered by 1/4 ML of Co adatoms. (e) $\mathrm{Cu}$ slab covered by $1 / 4 \mathrm{ML}$ of Co adatoms on a Co substrate. (f) Comparison of (d) (open circles) and (e) (closed circles). The data of (d) are offset by two layers along the abscissa and by $0.009 \mathrm{eV}^{-1}$ along the ordinate.

scales characteristic of the $\mathrm{Cu}$ Fermi surface: the distance between the "belly" points as well as the distance between the "neck" points. ${ }^{21}$ Within the framework of the phase accumulation model, the DOS at the surface of $\mathrm{Cu}$ layers varies according to the following equation: ${ }^{13,21}$

$$
\rho_{F}=\rho_{F, 0}+\sum_{i=b, n} \frac{A_{i}}{N_{\mathrm{Cu}}} \cos \left(\frac{2 \pi N_{\mathrm{Cu}}}{\Lambda_{i}}+\Phi_{i}\right) .
$$

Here, $\rho_{F}$ is the DOS at $E_{F}$ at the top Cu layer in the slab, and $\rho_{F, 0}$ is $\rho_{F}$ in the limit of $N \rightarrow \infty . A_{i}, \Lambda_{i}$, and $\Phi_{i}$ are the amplitudes, spatial periodicities, and phases of the quantum oscillations at the belly $(i=b)$ and the neck $(i=n)$ of the $\mathrm{Cu}$ Fermi surface, respectively. One may judge whether the electronic states in a model slab are well described by the phase accumulation model by fitting Eq. (1) to the ab initio result. While the periodicities are uniquely determined by the shape of the $\mathrm{Cu}$ Fermi surface $\left(\Lambda_{b}=5.88 \mathrm{ML}\right.$ and $\Lambda_{n}$ $=2.67 \mathrm{ML}),{ }^{9,21}$ the amplitudes and the phases are sensitive to the details of the electron reflection at the interfaces. Therefore, we have performed the fit keeping $\Lambda_{i}$ fixed and leaving $A_{i}$ and $\Phi_{i}$ as free parameters. To account for the background change in the calculated DOS, a linear term of $B N_{\mathrm{Cu}}$ was added to Eq. (1). This change is probably caused by incommensurability between $N$ and $\Lambda_{i}$ and is only an artifact. A reasonably good fit is obtained for $A_{b}$ $=0.039 \mathrm{eV}^{-1}, A_{n}=0.092 \mathrm{eV}^{-1}, \Phi_{b}=0.87 \pi$, and $\Phi_{n}=1.39 \pi$ [dashed line in Fig. 1(a)]. This confirms the interpretation of the DOS variation in terms of the phase accumulation model. Note that this model generally holds at least down to $N=5,{ }^{7,20}$ although Eq. (1) is mathematically exact only for $N \rightarrow \infty$.

In experiments ${ }^{13}$ the $\mathrm{Cu}(100)$ layers are supported by $\mathrm{Co}(100)$ layers, but the addition of Co layers does not change the above conclusion. Figure 1(b) shows the DOS at $E_{F}$ calculated for $\mathrm{Cu}$ slabs on a $\mathrm{Co}$ substrate (solid line). A similar oscillatory behavior of the DOS is visible, while its details are different from those of pure $\mathrm{Cu}$ slabs. The dashed line in Fig. 1(b) shows the result of the same fitting analysis using Eq. (1). Again, a reasonably good fit is obtained, with different parameters of $A_{n}=0.18 \mathrm{eV}^{-1}$ and $\Phi_{n}=0.01 \pi$. The oscillation at the belly is too small to be determined. To clarify the effect of the Co substrate, the data in Fig. 1(a) (open circles) and Fig. 1(b) (closed circles) are replotted in Fig. 1(c). Note that the data in Fig. 1(b) are offset by one layer along the abscissa and by $-0.018 \mathrm{eV}^{-1}$ along the ordinate. The very similar DOS variations in Figs. 1(a) and 1(b) indicate that the main role of the Co substrate is to change the phase shift at the interface. Consequently, the phase accumulation model remains valid for describing the electronic states.

The situation is drastically changed by adding only a fraction of a Co monolayer on top of the $\mathrm{Cu}$ surface. Figure 1(d) shows the calculated DOS at $E_{F}$ at the top $\mathrm{Cu}$ layer in $\mathrm{Cu}$ 


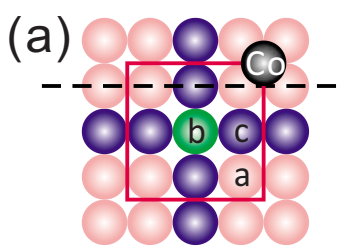

layer number $=$ odd

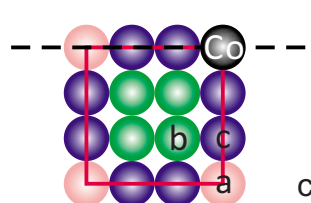

layer number = even

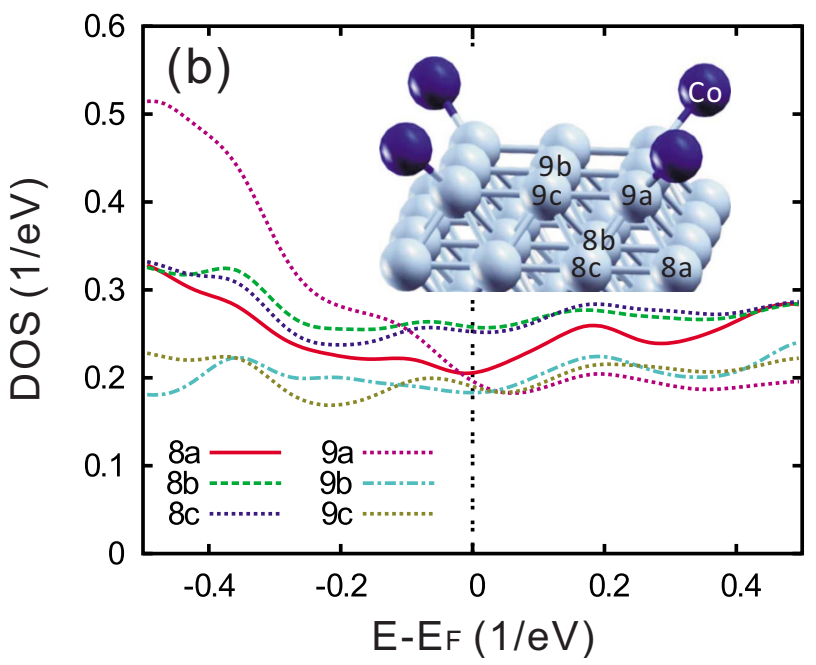

FIG. 2. (Color online) (a) Atomic arrangement used for the calculations and naming convention of columns a-c and layer numbers. Left panels: top views of the $\mathrm{Cu}(100)$ layers at odd and even layer numbers. Right panel: side view of $\mathrm{Cu}$ layers, which corresponds to the dashed lines in the top views. (b) DOS as a function of energy at the atomic sites labeled in the inset. Inset: model of the surface of a slab of nine $\mathrm{Cu}$ layers $\left(N_{\mathrm{Cu}}=9\right)$ with a $1 / 9 \mathrm{ML}$ of $\mathrm{Co}$ adatoms on top. slabs covered by 1/4 ML of Co adatoms (solid line). Note that the atomic arrangement is exactly the same as used in Fig. 1(a) except for the Co adatoms. An obvious change from Fig. 1(a) is that the oscillation with a periodicity of about 2.5 atomic layers is suppressed for $N_{\mathrm{Cu}}>10$. The calculated DOS cannot be properly fitted using Eq. (1) (dashed line; $A_{b}=0.042 \mathrm{eV}^{-1}, A_{n}=0.055 \mathrm{eV}^{-1}, \Phi_{b}=1.60 \pi$, and $\Phi_{n}=$ $-0.01 \pi)$. This failure of the phase accumulation model suggests that the QWSs are substantially perturbed by the presence of Co adatoms, which - in contrast to a complete monolayer-do not constitute a smooth interface. The addition of a Co substrate for simulating the experimental setup leads to the same conclusion as shown in Fig. 1(e) (solid line: $a b$ initio calculation; dashed line fitting result with $A_{b}$ $=0.11 \mathrm{eV}^{-1}, A_{n}=0.11 \mathrm{eV}^{-1}, \Phi_{b}=0.10 \pi$, and $\left.\Phi_{n}=-0.25 \pi\right)$. Figure 1(f) replots the data in Fig. 1(d) (open circles) and Fig. 1(e) (closed circles). Note that the data in Fig. 1(e) are offset by two layers along the abscissa and by $0.009 \mathrm{eV}^{-1}$ along the ordinate. Again, the similar behavior of the DOS variations suggests that the Co substrate causes a phase shift of electron waves. However, the strong perturbation of the DOS oscillations by a submonolayer coverage of Co adatoms is clear in the presence of a Co substrate, too. These observations call for investigations of the DOS at individual atomic sites in the $\mathrm{Cu}$ layers next to Co adatoms.

To observe possible atomic-scale variations of the DOS in the lateral direction, the unit cell was expanded to a $3 \times 3$ supercell (solid squares), which reduces the density of $\mathrm{Co}$ adatoms to 1/9 ML. Figure 2(a) shows a sketch of the atomic arrangement used for the calculations. The two figures on the left side show top views of the $\mathrm{Cu}(100)$ layers at odd and even layer numbers. The figure on the right side shows a side view of the $\mathrm{Cu}$ layers, which corresponds to the dashed lines in the top views. Figure 2(a) also introduces our naming convention of columns a-c and layer numbers $N_{\text {layer }}$ Co adatoms are placed on the top hollow site of column a while columns $\mathrm{b}$ and $\mathrm{c}$ are off the Co adatoms in lateral directions. Figure 2(b) displays the DOS as a function of energy at different atomic sites for $N_{\text {layer }}=8,9$ (see the inset). For both layers, atomic sites in columns b and c exhibit a similar

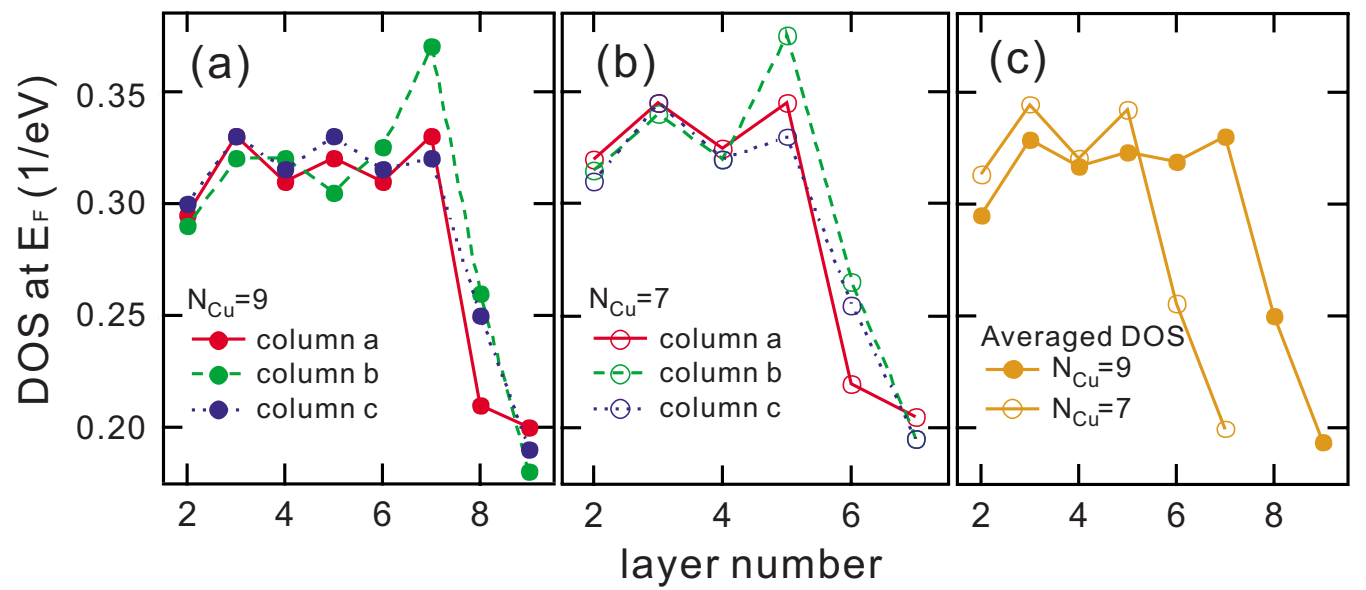

FIG. 3. (Color online) DOS at $E_{F}$ within columns a-c as a function of layer number. (a) $N_{\mathrm{Cu}}=9$. (b) $N_{\mathrm{Cu}}=7$. (c) DOS at $E_{F}$ averaged within each layer for $N_{\mathrm{Cu}}=7$, 9. DOSs at columns a-c are weight averaged according to their occupation numbers within a unit cell. 
energy dependence. In contrast, the variation of the DOS at atomic sites in column a is substantially different. This indicates that the effect of Co adatoms on the QWS varies on an atomic scale in a lateral direction.

The presence of laterally inhomogeneous QWS is confirmed by observing the oscillatory behavior of the DOS within different columns. The DOSs at $E_{F}$ were calculated for the same atomic arrangement as depicted in Fig. 2(a). Figure 3(a) displays the result for $N_{\mathrm{Cu}}=9$ as a function of $N_{\text {layer }}$ at columns a-c. Each column exhibits different DOS variations, demonstrating that the atomic-scale inhomogeneity of the QWS is indeed induced by the Co adatoms. Particularly, column $b$, which is off the Co adatoms, shows an opposite trend of the DOS variation as compared to the other two columns next to the center of the slab. Figure 3(c) shows the DOS at $E_{F}$ averaged within each layer (closed circles). The contributions from columns a-c were weighted according to their occupation numbers within a unit cell. Obviously, the oscillation in the averaged DOS is suppressed as compared to the individual columns. The spatial coherence, however, depends on the number of $\mathrm{Cu}$ layers. For $N_{\mathrm{Cu}}=7$, columns a-c exhibit similar DOS variations [see Fig. 3(b)].
Consequently, variation in the averaged DOS is more pronounced for $N_{\mathrm{Cu}}=7$ [open circles in Fig. 3(c)] than for $N_{\mathrm{Cu}}$ $=9$. The suppression of DOS oscillations for $N_{\mathrm{Cu}}>10 \mathrm{ob}-$ served in Figs. 1(d) and 1(e) may be attributed to this $\mathrm{N}_{\mathrm{Cu}^{-}}$ dependent spatial coherence.

In summary, the influence of Co adatoms on the DOS of QWSs in $\mathrm{Cu} / \mathrm{Co}(100)$ multilayers was investigated by means of $a b$ initio electronic structure calculations. The DOSs at $E_{F}$ of the surface $\mathrm{Cu}$ layer as a function of $N_{\mathrm{Cu}}$ are found to be strongly perturbed by a $1 / 4 \mathrm{ML}$ of Co adatoms. This makes an analysis in terms of the conventional phase accumulation model inadequate. The spatially resolved DOS in $\mathrm{Cu}$ slabs with a 1/9 ML of Co adatoms reveals atomic-scale variations of the QWS in a lateral direction. The spatial coherence of the QWS depends on $N_{\mathrm{Cu}}$, which can explain the drastic change in the DOS variation as a function of $N_{\mathrm{Cu}}$ due to the Co adatoms.

T.U. acknowledges financial support from JSPS Grant-inAid for Scientific Research Grant No. 21510110 from the Japanese Society for the Promotion of Science. R.B. acknowledges SFB 668 for financial support. *udo.schwingenschlogl@kaust.edu.sa

${ }^{1}$ M. N. Baibich, J. M. Broto, A. Fert, F. Nguyen Van Dau, F. Petroff, P. Etienne, G. Creuzet, A. Friederich, and J. Chazelas, Phys. Rev. Lett. 61, 2472 (1988).

${ }^{2}$ Y. Arakawa and H. Sakaki, Appl. Phys. Lett. 40, 939 (1982).

${ }^{3}$ U. Schwingenschlögl and C. Schuster, EPL 81, 17007 (2008); 86, 27005 (2009).

${ }^{4}$ P. M. Echenique and J. B. Pendry, J. Phys. C 11, 2065 (1978).

${ }^{5}$ N. V. Smith, Phys. Rev. B 32, 3549 (1985).

${ }^{6}$ M. Milun, P. Pervan, and D. P. Woodruff, Rep. Prog. Phys. 65, 99 (2002).

${ }^{7}$ J. E. Ortega and F. J. Himpsel, Phys. Rev. Lett. 69, 844 (1992); J. E. Ortega, F. J. Himpsel, G. J. Mankey, and R. F. Willis, Phys. Rev. B 47, 1540 (1993).

${ }^{8}$ T. C. Chiang, Surf. Sci. Rep. 39, 181 (2000).

${ }^{9}$ Z. Q. Qiu and N. V. Smith, J. Phys.: Condens. Matter 14, R169 (2002).

${ }^{10}$ A. A. Khajetoorians, G. A. Fiete, and C.-K. Shih, Phys. Rev. B 81, 041413(R) (2010).

${ }^{11}$ J. Kliewer, R. Berndt, and S. Crampin, Phys. Rev. Lett. 85, 4936 (2000).

${ }^{12}$ O. O. Brovko, P. A. Ignatiev, V. S. Stepanyuk, and P. Bruno, Phys. Rev. Lett. 101, 036809 (2008).

${ }^{13}$ T. Uchihashi, J. Zhang, J. Kröger, and R. Berndt, Phys. Rev. B 78, 033402 (2008).
${ }^{14}$ X. Zhang, A. Zhao, K. Wang, and X. Xiao, Phys. Rev. B 78, 035431 (2008).

${ }^{15}$ Y.-S. Fu, S.-H. Ji, X. Chen, X.-C. Ma, R. Wu, C.-C. Wang, W.-H. Duan, X.-H. Qiu, B. Sun, P. Zhang, J.-F. Jia, and Q.-K. Xue, Phys. Rev. Lett. 99, 256601 (2007).

${ }^{16}$ P. van Gelderen, S. Crampin, and J. E. Inglesfield, Phys. Rev. B 53, 9115 (1996).

${ }^{17}$ U. Schwingenschlögl and I. A. Shelykh, Phys. Rev. B 80, 033101 (2009).

${ }^{18}$ C. M. Wei and M. Y. Chou, Phys. Rev. B 68, 125406 (2003).

${ }^{19}$ Y. Han and D.-J. Liu, Phys. Rev. B 80, 155404 (2009).

${ }^{20}$ J. M. An, D. Raczkowski, Y. Z. Wu, C. Y. Won, L. W. Wang, A. Canning, M. A. Van Hove, E. Rotenberg, and Z. Q. Qiu, Phys. Rev. B 68, 045419 (2003).

${ }^{21}$ P. Bruno, Phys. Rev. B 52, 411 (1995); J. Phys.: Condens. Matter 11, 9403 (1999).

${ }^{22}$ V. Eyert, The Augmented Spherical Wave Method: A Comprehensive Treatment, Lecture Notes in Physics Vol. 719 (Springer, Heidelberg, 2007).

${ }^{23}$ S. H. Vosko, L. Wilk, and M. Nusair, Can. J. Phys. 58, 1200 (1980).

${ }^{24}$ P. Alippi, P. M. Marcus, and M. Scheffler, Phys. Rev. Lett. 78, 3892 (1997).

${ }^{25}$ A. B. Shick, D. L. Novikov, and A. J. Freeman, Phys. Rev. B 56, R14259 (1997). 